\title{
Alveolar corticotomies by lasercision: a minimally invasive procedure to accelerate orthodontic treatments in adult patients
}

\author{
B. Savard \\ DDS in dental surgery - Private Practice
}

SUMMARY

In our offices, more and more adults are visiting with the wish to align their teeth to preserve the aesthetics of their smile. However, treatment duration is the most common reason in the refusal to undergo orthodontic treatment. Alveolar corticotomies help accelerate and facilitate tooth movement. This is a surgical technique that consists of vertical scarifications of the vestibular interdental cortical bone to stimulate the alveolar bone metabolism. Current protocols, thanks to recent studies on bone physiology, are much less invasive than before and they allow more frequent indications for use of this technique, to meet the demands in adults.

\section{KEYWORDS}

Alveolar corticotomies, accelerated orthodontics, rapid orthodontics, lasercision

In recent years, the demand for orthodontic treatments in adult patients has been steadily increasing. The smile has a major impact on self-image and alterations in the smile are a sign of aging. In a society where staying young and healthy for as long as possible is universally desired, having "healthy, aligned teeth" is a recurring request. In addition, people are now aware of the importance of having aligned teeth and functional occlusion, not only for aesthetic reasons but to correct functional issues such as periodontal risk, joint disorders, and carious lesions. Finally, general practitioners are requesting preprosthetic orthodontic treatments with increasing frequency.
However, adult patients are often reluctant to engage in orthodontic treatment, which, despite the inconspicuous nature of its techniques, remains restrictive in terms of treatment duration, lifestyle disruption, appointment frequency, speech difficulties, surgical wounds, and pain.

As a result, we are often confronted with patients who will refuse orthodontic treatment, especially because of treatment duration, and who prefer that their smile be rehabilitated by prosthetic means.

Alveolar corticotomies make it possible to decrease the duration of an orthodontic treatment and to facilitate treatment progression. It is a surgical technique that

Address for correspondence:

Brice Savard

24, rue Boissière - 75116 Paris - France

E-mail: dr.savard@cliniquedentaireboissiere.fr

Article received: 21-04-2016. Accepted for publication: 06-06-2016.

This is an Open Access article distributed under the terms of the Creative Commons Attribution License (http://creativecommons.org/licenses/by/4.0), which permits unrestricted use, distribution, and reproduction in any medium, provided the original work is properly cited. 
consists of performing vertical scarifications of the vestibular interdental cortical bone in the maxilla and the mandible so as to accelerate dental movements. In recent years, less-invasive procedures have been developed, which can be more easily integrated into our treatment plans.

We will describe a technique of minimally invasive alveolar corticotomies, which are deemed laser-assisted as they use lasercision.

\section{BACKGROUND}

The first protocol was described by Köle ${ }^{9}$ in 1959, associating vestibular and lingual segmental corticotomies and a supra-apical horizontal osteotomy after the elevation of a complete subperiosteal flap. Köle proposes the bony blocks theory to explain faster dental displacements-the theory states that it is necessary to obtain an almost complete dissociation of the bone block supporting the alveolodental processes so that the movement is more rapid. This technique was extremely invasive. Simpler, less-invasive protocols will be developed ${ }^{1,5,6}$ according to the bone block theory.

In 1983, Frost, an American orthopedic surgeon, introduced the concept of the regional activation phenomenon $(\mathrm{RAP})^{3,4}$. As a result of surgical procedures on the long bones, there is an increase in the rate of bone repair (bone remodeling) associated with a state of transient osteopenia in the immediate vicinity of the intervention site, leading to a faster physiological healing process.

Many studies have validated this concept and correlations have been made between postoperative RAP and dental mobility. Yaffe et al. ${ }^{19}$ propose that RAP is responsible for increasing dental mobility after periodontal surgery. Verna ${ }^{16}$ shows a correlation between bone remodeling and dental displacement.

\section{THE BIOLOGICAL CONCEPT OF CORTICOMIES}

On the basis of the RAP theory, the Wilcko brothers (an American orthodontist and a periodontist) introduced the biological concept in 2001 to explain the increase in the rate of dental displacement following corticotomies. ${ }^{18}$ The increased displacement speed is no longer merely the result of the mechanical embrittlement of the cortical bone but is the result of a decrease in bone density near the intervention site and an increase in the upregulation of the osteoblast-induced bone turnover.

However, their protocol is always conventional and invasive as it comprises a complete mucoperiosteal flap, in the vestibular and lingual areas, followed by osteotome-based corticotomies (possibly associated with a soft and/or bony tissue graft).

Studies of RAP have shown that the effect of decreasing bone density is 
found at a distance from the corticotomy sites ${ }^{10-13,17}$, and less-invasive, flapless protocols have been successfully developed ${ }^{78}$. Wang ${ }^{17}$ and Lee ${ }^{10}$ demonstrate that RAP is responsible for accelerated dental movements.

In 2009, Dibart, Sebaoun, and Surmenian $^{2}$ introduced the piezocision technique to perform minimally invasive segmental corticotomies. This innovative technique, based on the theory of RAP, eliminated the need for the mucoperiosteal flap and palatal corticotomies. Corticotomies are now performed using a piezotome, directly through the attached gingiva, after a vertical incision is placed using a bistoury. This incision on the cortical bone is approximately $2 \mathrm{~mm}$ deep.

This technique can be combined with soft-tissue and/or bone grafts. The locoregional action of RAP allows for a bone density reduction around the teeth near the corticotomy.

\section{INDICATIONS AND CONTRAINDICATIONS OF CORTICOMIES}

The goal of corticotomies is to significantly decrease the duration of orthodontic treatment and to facilitate its progression.

They can be performed in most malocclusions. Corticotomies will be particularly useful in the dental leveling phase, for example when treating a class-I DMD. In this case, the corticotomies are performed at the beginning of the treatment on both arches in their entirety. The duration of RAP is shorter, approximately 6 months.

However, corticotomies are usually found in more complex treatments. Corticotomies will mild and/or severe dental displacements (maxillary or mandibular molar distalization, axial straightening, intrusions etc.), while decreasing the risk of rhizolysis and confounding effects which may be caused by a localized weakening of the relative anchorage. Corticotomies will thus simplify the treatment plan and decrease the use of orthodontic extractions or surgical treatments.

The clinical case presented is an example of the use of corticotomies in a localized manner at the different phases of a complex treatment.

Corticotomies are also indicated in the decompensation phase during orthodontic surgical treatments.

On the other hand, corticotomies can be performed regardless of the orthodontic technique used (vestibular, lingual, aligners). The bows are changed every 2 weeks, whereas the aligners must be changed weekly.

Patients undergoing this procedure should not have active periodontal disease, periapical lesions, local or general bone disease, immunosuppressive therapy, bisphosphonates, or corticosteroids (changes in cell regeneration). Patients should not have a risk of infectious endocarditis and a history of radiotherapy in the head and neck areas. Smokers have an increased risk of postoperative infection.

Beforehand, a periodontal and dental assessment will be carried out. All carious lesions, periapical abscesses, and active periodontal disease will have to be dealt with before initiating orthodontic treatment. Descaling, possibly 
accompanied by a root planning, will be performed shortly before surgery.

Finally, it is essential to perform a three-dimensional cone-beam computed tomography of the maxilla and the mandible to identify the root axis and root proximities, measure the cortical thickness and, and visualize the mental foramen.

\section{LASER-ASSISTED CORTICOMY: LASERCISION}

The erbium laser has been used successfully for many years to perform precise bone and mucogingival surgeries (sinus lift, coronary elongations, surgical endodontics, periodontal surgery, etc.).

There are two types of erbium lasers: the Er:YAG laser (whose active medium is composed of yttrium crystal, aluminum, garnet enriched with erbium, wavelength $\lambda=2940 \mathrm{~nm}$ ) and the laser Er, Cr:YSGG (yttrium crystal, scandium, gallium, garnet, enriched with erbium and chromium, $\lambda=2,780 \mathrm{~nm}$ ).

It is important to remember that the erbium laser is the only laser used in odontology allowing surgical action on bony tissue.

In 2012, Seifi, Younessian, and Ameli15 have demonstrated that in corticotomies performed on beagle dogs, substituting the flap for an erbium laser (Er, Cr: YSGG, Waterlase, Biolase, USA) created accelerated dental displacement. The ablative effect of the erbium laser on the cortical bone causes a RAP-type response, without postoperative sequelae or aftereffects.

We are proposing a technique involving minimally invasive corticotomies, without the mucoperiosteal flap, using the erbium laser: Lasercision.

\section{Principle of operation of the erbium laser}

The wavelengths of erbium lasers $(2,940$ and $2,780 \mathrm{~nm})$ are strongly absorbed by water and hydroxyapatite. The energy of the pulse transmitted to the water molecules will cause instantaneous and localized vaporization (5- $\mu \mathrm{m}$ depth). The sudden rise in intratissue pressure will cause a microexplosion (known as explosive vaporization) and dissociation of cells from the target tissue. This is the photoablative effect. There is also a direct but attenuated action on hydroxyapatite (a photomechanical effect). Because of the high energy absorption by water, there is no carbonization of the target tissue. In addition, there is no remote thermal effect, which precludes the risk of tissue necrosis. Because the ablation is localized ( $5-\mu \mathrm{m}$ deep), an extremely fine incision can be made.

\section{Clinical procedure when using the erbium laser}

The procedure is performed a few days after the orthodontic brackets are placed, or even the same day when transparent aligners are used.

LightWalker ${ }^{\circledR}$ AT (Fig. 1) from Fotona (Ljubljana, Slovenia) is used in the Er:YAG laser $(\lambda=2,940 \mathrm{~nm})$. The power used is $2 W$ at the attached gingiva (energy $=200 \mathrm{~mJ}$, frequency of pulses $=10 \mathrm{~Hz}$, mode MSP + water and airsprays) then $3 \mathrm{~W}$ at the level of the bone (energy = $200 \mathrm{~mJ}$, frequency of the pulses $=15$ $\mathrm{Hz}$, mode QSP + sprays of water and air). 
These powers are sufficient to allow a rapid gingival and bone ablation without risking thermal damage.

Local anesthesia is administered beforehand. We use a beveled tip allowing an extremely fine incision of the gum and the bone. The procedure is performed only in the vestibular area. We penetrate directly with the tip through the attached gingiva until reaching the cortical bone. The tip must always have an angulation of between $45^{\circ}$ and $60^{\circ}$ in relation to the surface. The tip is used in the precontact phase. Avoid touching the gums and cortical bone.

It is advisable to start the incision at the mucogingival junction and to go up to the level of the interdental papilla without damaging it. Several passages are necessary. It is recommended to "sweep" the surface by starting each time from the starting point, so as not to shred the gum with the tip.

We then perform an ablation of the alveolar bone between each tooth that we wish to move, following the same procedure as for the attached gingiva (sweeping from apical to coronal aspects so as not to touch the cortex with the tip). The power and frequency of the pulses are increased.

The depth of intracortical penetration is approximately $2-3 \mathrm{~mm}$, depending on the cortical thickness measured with the cone beam. In all cases, the bone cortex should not be crossed. We measure the depth of penetration using a graduated periodontal probe. It is important to stay in the attached gingiva and not incise the highly vascularized mucosal area. However, it is possible, using the tip, to penetrate under the mucosa to reach the cortical bone, to achieve a more extended apical corticotomy. This is systematically done.

A gingival and / or bone graft may be associated in areas of low cortical thickness, by peeling the gingiva between each incision, as described by Sebaoun, Surmenian, and Dibart.

At the end of the operation, hemostasis is obtained using sterile compresses. No suturing is necessary and gingival healing is very rapid. No antibiotic therapy is prescribed (because of the bactericidal effect of the laser). We give an analgesic, NSAID or paracetamol to the patient at the end of the procedure. Most of the time, the patient feels no postoperative pain, only a tingling sensation (analgesic effect of lasers). No swelling or edema occurs (anti-inflammatory effect of lasers). The patient can return to work the same day.

We review the patient one week after the operation. Orthodontic arches are changed every 15 days (instead of 4-6 weeks). Aligners are changed weekly (instead of 2-3 weeks).

\section{The advantages of using the erbium laser for corticotomies}

The advantages of the laser compared to the conventional techniques are numerous:

- As the laser is used in "the precontact phase," there is no unpleasant sensation for the patient during the intervention (no vibration).

- No postoperative pain, nor edema (analgesic and anti-inflammatory effect of the laser by the phenomenon of biomodulation).

- No heating (air spray and water) and thus no risk of tissue necrosis. 
- Gingival regeneration is achieved very quickly and without scarring (a biomodulation effect on the gingival healing process).

- Using a fine tip, it is possible to carry out a more extensive corticotomy under the mucous membrane without damaging it.

\section{CONCLUSION}

Alveolar corticotomies by lasercision are reliable and very well tolerated by patients, with the advantages inherent in lasers.

With the development of minimally invasive procedures (piezocision and lasercision), alveolar corticotomies must now be integrated into orthodontic treatment

\section{THE CLINICAL CASE}

She is a 26-year-old patient who has been displeased with her smile for many years, especially with the ectopic position of her right maxillary canine. She refuses to smile because of her teeth. She has never received orthodontic treatment. She plans to follow a course of treatment to correct her malocclusion and regain a harmonious smile. She is reluctant to wear brackets neither externally nor internally and is desirous of a rapid, nonrestrictive treatment.

\section{Diagnosis and treatment}

The patient displayed a class-II malocclusion on the right side and a class I on
- The speed of execution with a power of approximately $3 \mathrm{~W}$.

- Biostimulation phenomenon specific to all lasers (Low-Level Laser Therapy); studies show an effect on the acceleration of dental displacement. plans for adults. When being confronted with complex situations, alveolar corticotomies will allow the simplification of therapy and development of optimal treatment plans. In addition, by promoting faster treatments, adult patients will be much more tolerant of the discomfort associated with treatment.

the left (class-III limit), with an asymmetry of the middle interincisors caused by a shift of the lower interincisor midline to the right. The patient had a minor skeletal class-II malocclusion with slight brachyfacial typology.

The MDD is $-9 \mathrm{~mm}$. The right maxillary canine 13 is in the vestibular position with mesialization of sector 1 . Similarly, the right mandibular canine 43 is in vestibular position with mesialization of sector 4. The mandibular incisors are vestibuloversed.

Esthetically, the patient possesses a harmonious profile.

Despite the mandibular overcrowding and the vestibuloversion of the lower 


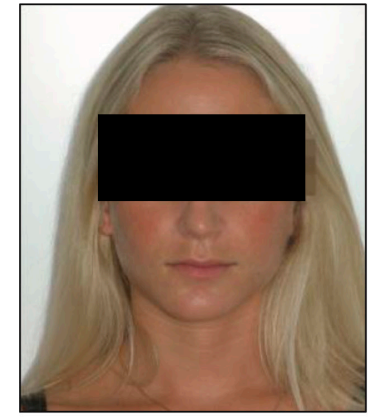

a

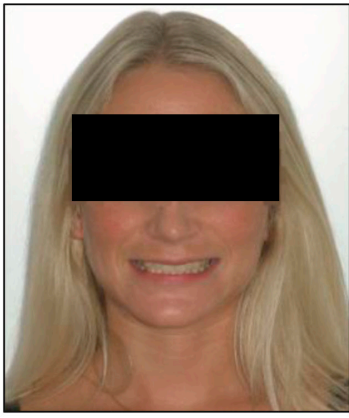

b

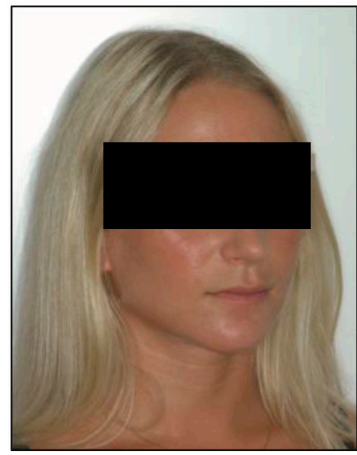

e

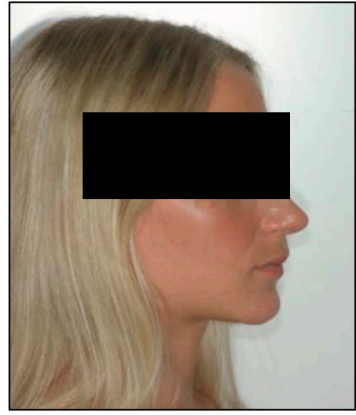

C

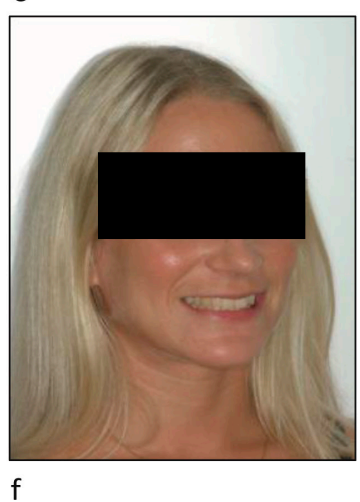

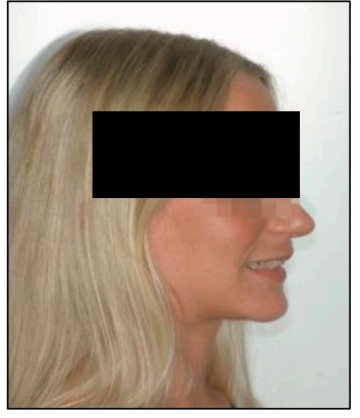

Figure $1 a, b, c, d, e, f$

Extraoral photographs at the start of treatment.

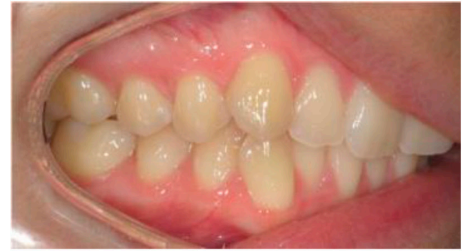

a

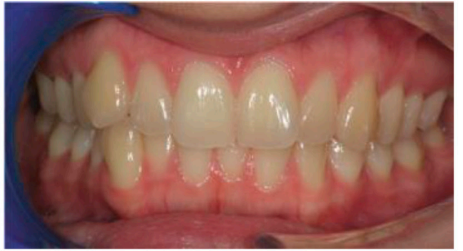

b

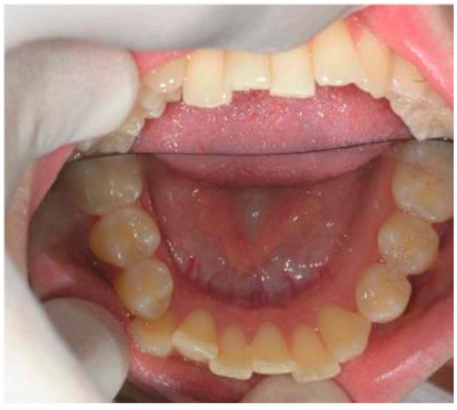

d

e

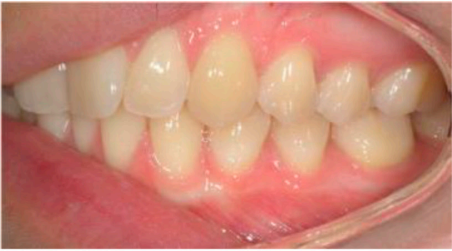

c

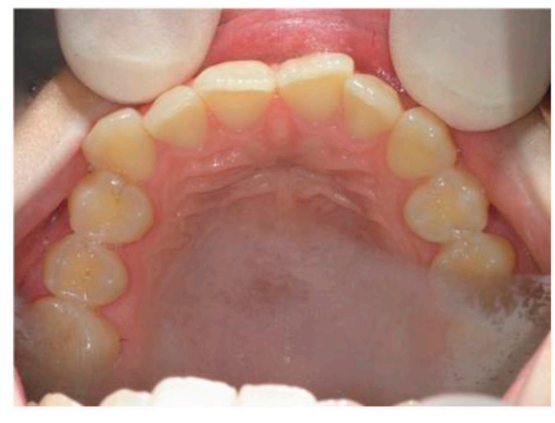

Figure 2a, b, c, d, e

Intraoral photographs at the start of treatment. 

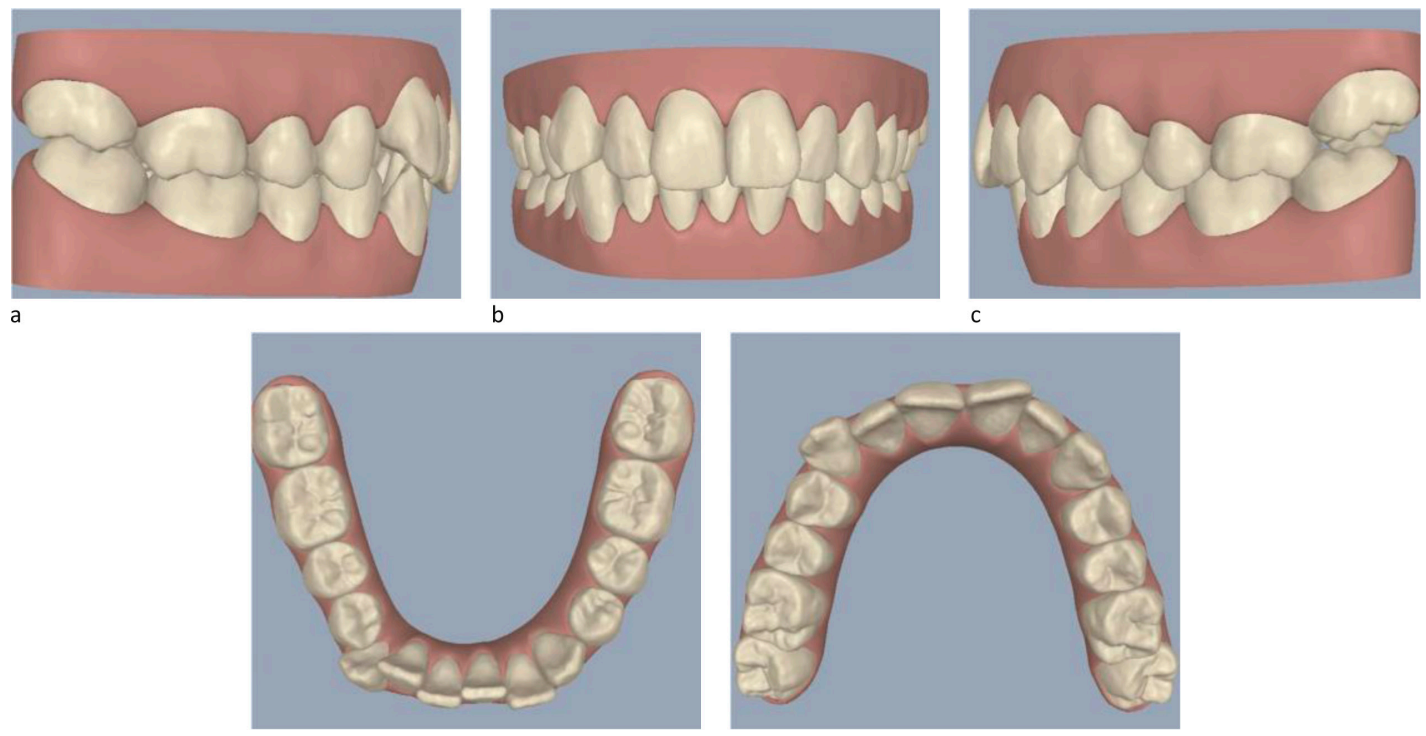

Figure $3 a, b, c, d$, e

Optical impression images at the start of treatment.
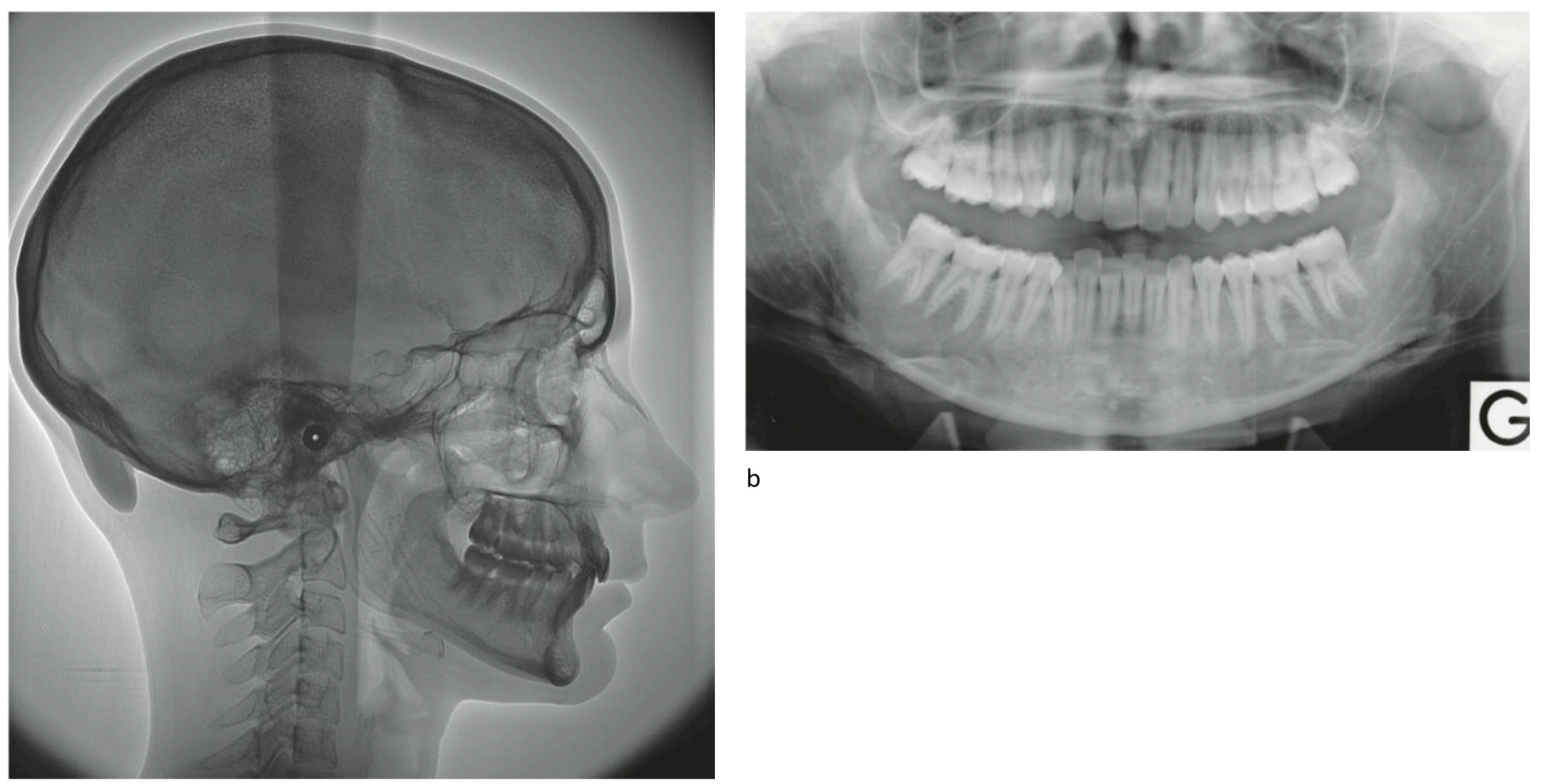

b

Figure $4 a, b$

Initial radiographs. 
incisors, and given the age and profile of the patient, we wished to perform treatment without extraction. The amount of stripping required for repositioning the incisors was far too significant to be considered. We chose a treatment with distalization of sectors 1 and 4 so as to be able to reposition the canines and limit the lower interproximal reductions. Analysis of the profile radiograph showed an increased 6-Ptv distance,

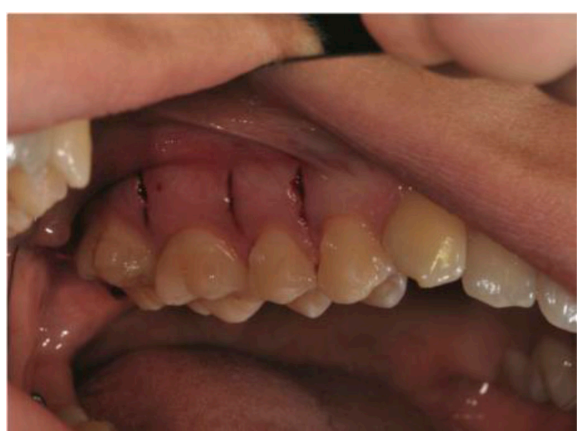

Figure 5

Corticotomies of sector 1.

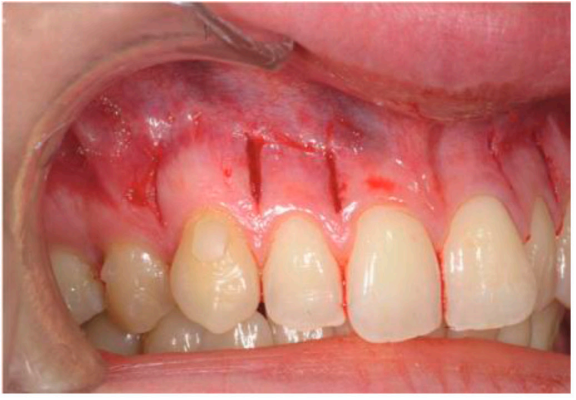

a

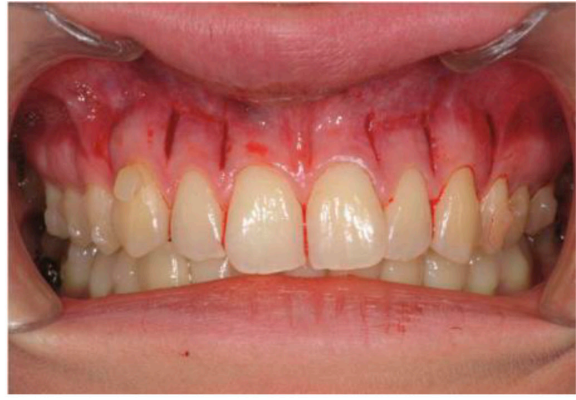

b

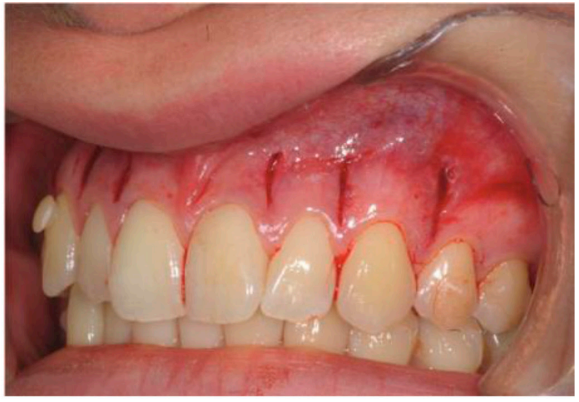

C

Figure $6 a, b, c$

Anterior maxillary cortico tomies.

absence of the 3rd molars, with space available distal to 7 .

The patient was planned to receive aligner treatment. To facilitate the initial distalization stage, we decided to perform segmental corticotomies via the sequencing of the sectors 1 and 4 , from the distal face of 7 to the mesial face of 5 . Sectors 7, 6, 5, and 4 were moved one by one. The other teeth are not subjected to corticotomies to reinforce the anchorage. Once the distalization is achieved, we proceeded to the recoil of 13 and 43 (facilitated by direct elastic traction on these teeth), which was followed by incisor-canine leveling.
Before this, segmental corticotomies were performed by lasercision of the maxillary and mandibular anterior segments (beginning distal to the canines). We chose not to perform corticotomies on the left side because the movements to be performed were of low amplitude, especially at the molar level.

We eventually completed the repositioning of the middle interincisors by intermaxillary tractions.

In total, the treatment lasted 10 months (with a distalization phase of about 5 months). The patient changed her aligners weekly.

Conflict of interest: The author has no conflict of interest. 


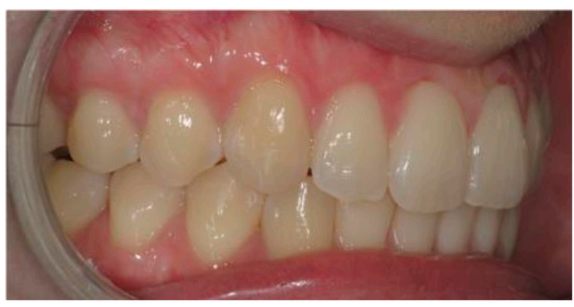

a

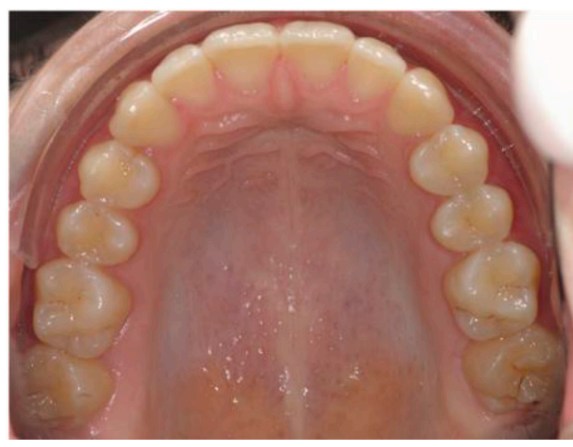

d

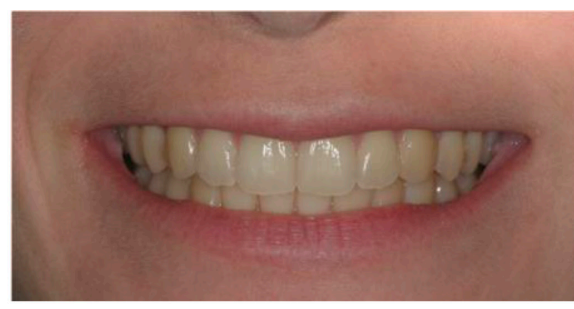

$b$

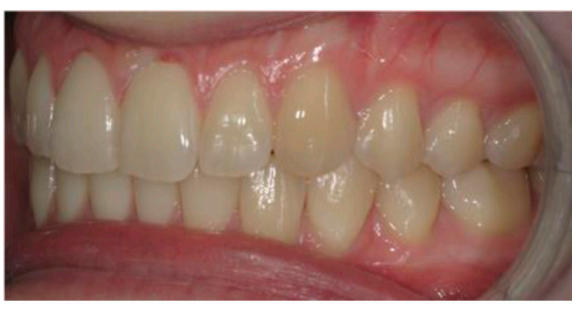

C

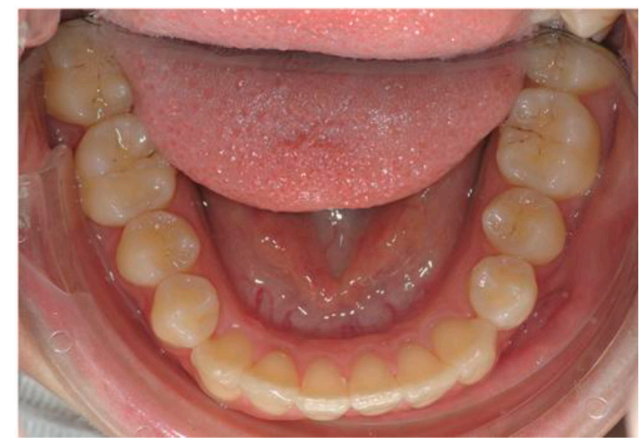

e

Figure $7 a, b, c, d, e$

Intraoral photographs at the end of treatment.

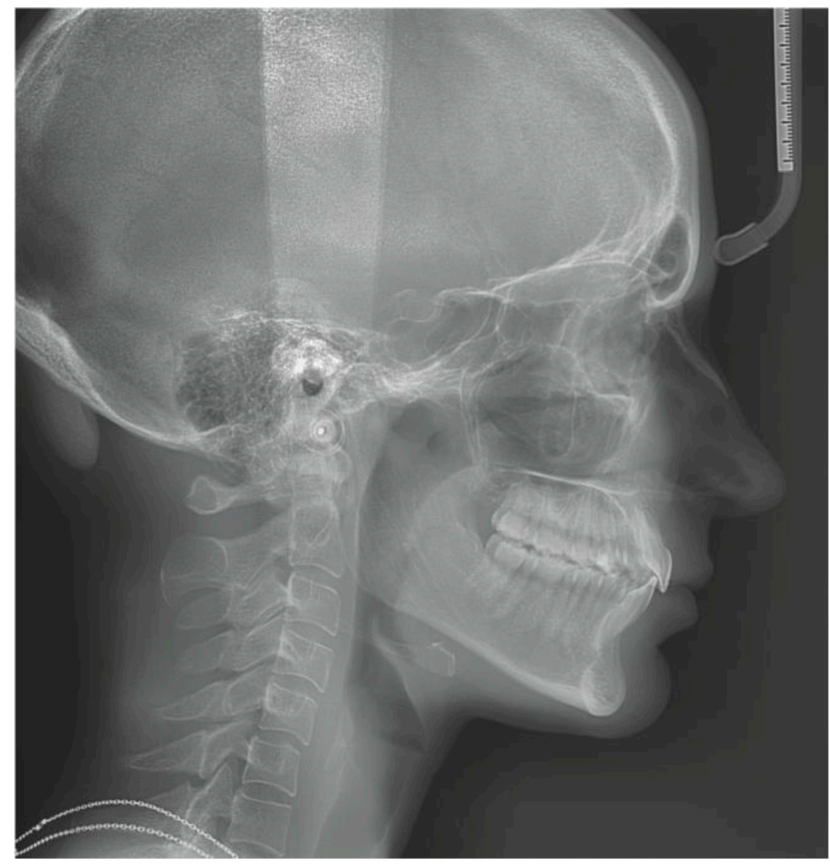

a

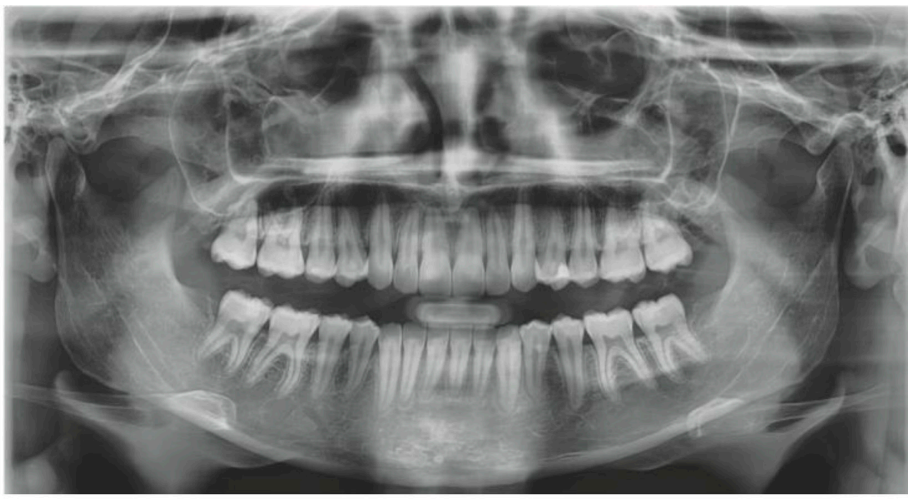

b

Figure $8 a, b$

Radiographs at the end of treatment. 


\section{BIBLIOGRAPHY}

1. Anholm JM, Crites DA, Hoff R, Rathbun WE. Corticotomy facilitated orthodontics. Calif Dent Assoc J 1986;14:7-11.

2. Dibart S, Sebaoun JD, Surmenian J. Piezocision: a minimally invasive, periodontally accelerated orthodontic tooth movement procedure. Compend Contin Educ Dent 2009;30:342-50.

3. Frost HM. The regional acceleratory phenomenon: a review. Henry Ford Hosp Med J 1983;31:3-9.

4. Frost HM. The biology of fracture healing. An overview for clinicians. Part II. Clin Orthop Relat Res 1989;248:294-309.

5. Gantes B, Rathbun E, Anholm M. Effects on the periodontium following corticotomyfacilitated orthodontics. J Periodontal 1990;61:234-238.

6. Generson RM, Porter JM, Zell A, Stratigos GT. Combined surgical and orthodontic management of anterior open bite using corticotomy. J Oral Surg 1978;36:216-219.

7. Germeç D, Giray B, Kocadereli I, Enacar A. Lower incisor retraction with a modified corticotomy. Angle Orthod 2006;76:882-890.

8. Kim SJ, Park YG, Kang SG. Effects of Corticision on paradental remodeling in orthodontic tooth movement. Angle Orthod 2009;79:284-291.

9. Köle H. Surgical operations of the alveolar ridge to correct occlusal abnormalities. Oral Surg Oral Med Oral Pathol 1959;12:515-529.

10. Lee $W$, et al. Corticotomy/ossteotomy-assisted tooth movement microCTs differ. J Dent Res 2008;87(9):861867.

11. Mostafa YA, Mohamed Salah Fayed M, Mehanni S, EIBokle NN, Heider AM. Comparison of corticotomy-facilitated vs standard tooth-movement techniques in dogs with miniscrews as anchor units. Am J Orthod Dentofacial Orthop 2009;136:570-577.

12. Ren A, LVT, Kang N, Zhao B, Chen Y, Bai D. Rapid orthodontic tooth movement aided by alveolar surgery in beagles. Am J Orthod Dentofacial Orthop 2007;131:160.e1-10.

13. Sebaoun JD, Kantarci A, Turner JW, Carvalho RS, Van Dyke TE, Ferguson DJ. Modeling of trabecular bone and lamina dura following selective alveolar decortication in rats. J Periodontol 2008;79:1679-1688.

14. Sebaoun JD, Surmenian J, Dibart S. [Accelerated orthodontic treatment with piezocision: a mini-invasive alternative to conventional corticotomies]. Orthod Fr 201;82:311-319.

15. Seifi M, Younessian F, Ameli N. The Innovated Laser Assisted Flapless Corticotomy to Enhance Orthodontic Tooth Movement. J Lasers Med Sci 2012;3(1):20-25.

16. Verna $C$, Dalstra M, Melsen B. The rate and the type of orthodontic tooth movement is influenced by bone turnover in a rat model. Eur J Orthod 2000;22:343-352.

17. Wang L, Lee W, Lei DL, Liu YP, Yamashita DD, Yen SL. Tisssue responses in corticotomy- and osteotomy- assisted tooth movements in rats: histology and immunostaining. Am J Orthod Dentofacial Orthop 2009;136:770.e1-11; discussion 770-771.

18. Wilcko WM, Wilcko T, Bouquot JE, Ferguson DJ. Rapid orthodontics with alveolar reshaping: two case reports of decrowding. Int J Periodontics Restorative Dent $2001 ; 21: 9-19$.

19. Yaffe A, Fine N, Binderman I. Regional accelerated phenomenon in the mandible following mucoperiosteal flap surgery. J Periodontal 1994;65:79-83. 